\title{
DISPOSITIVO DE MONITORIZACIÓN POSTURAL PARA PERSONAS DE LA TERCERA EDAD CON ALTO GRADO DE SEDENTARISMO
}

\author{
Patrick Vermander Nerea Perez Aitziber Mancisidor Itziar Cabanes \\ \{patrick.vermander, nerea.perez, aitziber.mancisidor, itziar.cabanes \}@ehu.eus \\ Departamento de Ingeniería de Sistemas y Automática, \\ Escuela de Ingeniería de Bilbao, UPV/EHU
}

\section{Resumen}

La monitorización postural en personas de la tercera edad es fundamental para evitar la aparición de problemas músculo-esqueléticos, así como la prevención de situaciones de riesgo y caídas. En este trabajo, se presenta un dispositivo de monitorización compuesto por 16 sensores FSR (Force Sensitive Resistor), colocados de forma discreta a lo largo del asiento y respaldo de una silla. La selección de la ubicación de estos sensores se ha llevado a cabo mediante un estudio previo, donde se han identificado los puntos más significativos. Para validar el dispositivo de monitorización, se han realizado diferentes ensayos experimentales donde se han comparado los resultados obtenidos mediante el dispositivo propuesto con los medidos con una malla de presión comercial. Los resultados muestran que las posturas presentadas quedan correctamente caracterizadas mediante el sistema propuesto, reduciendo el coste del sistema y aumentando su autonomía.

Palabras clave: Análisis Postural; Monitorización; Sedentarismo; Sensores de Fuerza.

\section{INTRODUCCIÓN}

A lo largo de las últimas décadas se ha producido un fenómeno de envejecimiento de la población a nivel mundial, donde las personas con una edad superior a 65 años suponen ya una décima parte de la población total [19]. En el caso de la población española, el número de mayores se ha duplicado en menos de 30 años [1]. Las perspectivas de futuro son aún más pesimistas, y sitúan en 2050 a España como el país más envejecido del mundo, debido entre otros factores, a la cada vez mayor esperanza de vida, y a la baja tasa de natalidad.

Junto con el aumento de la población envejecida y el impacto que ésta supone, aparece implícitamente un aumento de los comportamientos sedentarios [7]. Se estima que el $67 \%$ de la población mayor de 65 años pasa una media diaria de 8.5 horas en actitud sedentaria [18]. Esta actitud sedentaria afecta negativamente en el estado físico y mental de las personas mayores, empobreciendo su calidad de vida [4].

El debilitamiento muscular a causa del sedentarismo y la edad, puede provocar la adopción de malas posturas en sedestación [5]. Lo que puede llevar a generar dolores de espalda, hombros y cuello, tensión muscular, caídas y problemas nerviosos como ciática entre otros [21, 20]. Es por ello necesario, mantener una correcta salud postural con el fin de prevenir posibles trastornos musculo esqueléticos, especialmente en un colectivo físicamente tan vulnerable.

En la actualidad, dada la imposibilidad de que los especialistas sanitarios realicen un seguimiento continuado, la monitorización y diagnostico postural se lleva a cabo mediante cuestionarios específicos a rellenar por el paciente. Tratando de eliminar el componente subjetivo característico de estos cuestionarios, en los últimos años, ha crecido el interés entorno al desarrollo de dispositivos de monitorización postural que permitan cuantificar de forma objetiva el estado postural de un paciente. Estos dispositivos se pueden clasificar en tres grupos en función de cuan invasiva es la tecnología utilizada para la medición: sensores vestibles, sensores de visión y sensores de presión o fuerza.

Los dispositivos de monitorización basados en sensores vestibles hacen uso de sensores incorporados sobre la vestimenta o el propio cuerpo para recopilar información de una forma continua a lo largo del día. Dentro de este grupo, destaca la utilización de sensores inerciales IMU (Inertial Measurement Unit) para la medición de diferentes ángulos de desviación de la espina dorsal, tanto en el plano sagital como en el plano coronal $[5,6,8]$. Sin embargo, este tipo de sensores, además de resultar muy invasivos para el usuario, pueden sufrir interferencias debido al contacto entre el sensor y la ropa o el sensor y el cuerpo humano.

Es por ello, que otros autores han optado por la utilización de cámaras de visión para la monitorización postural $[2,16,12]$. Con el uso de las cámaras se busca hallar la localización de puntos de referencia del cuerpo humano que den idea de la disposición general de la postura adoptada. Pese 
a no tratarse de un sistema invasivo para el usuario, presenta una serie de limitaciones que hacen que su uso no proporcione la información suficiente para la caracterización postural. Entre estas limitaciones destacan su rango de acción limitado, así como las imprecisiones debidas a oclusiones en la imagen tomada.

En último lugar, se encuentran los sensores de presión o fuerza, que, ya sean conductivos en formato textil $[14,10]$ o resistivos $[13,11]$, se disponen a lo largo de la superficie de apoyo tanto en asiento como respaldo, permitiendo cuantificar la presión ejercida sobre ellos. Este tipo de sensores no son invasivos al estar situados sobre una superficie externa, y permiten una cierta portabilidad. Existen en la actualidad mallas de presión comerciales, que permiten monitorizar la actividad postural, como el sistema BPMS de Tekscan Inc [3] o el Seating Mat Dev Kit de Sensing Tex [17]. Sin embargo, estas soluciones disponen de un número excesivo de sensores, lo que hace que sean caros y de un tiempo de uso limitado. Además, al tratarse de soluciones cerradas, el almacenamiento y acceso de datos es limitado.

Ante esta situación, y con la idea de reducir el número de sensores utilizados, en este artículo se presenta un dispositivo de monitorización postural de bajo coste. En este dispositivo, con el fin de realizar una caracterización postural adecuada, se han colocado 16 sensores de fuerza FSR (Force Sensitive Resistor) en puntos significativos del asiento y respaldo de una silla.

El resto del artículo se organiza de la siguiente forma. En la sección 2, se realiza un análisis postural para detectar los puntos significativos donde se colocarán los sensores FSR. En la sección 3, se describe el dispositivo de monitorización postural desarrollado. En la sección 4, se detalla la validación del dispositivo. Por último, en la sección 5, se resumen las ideas más importantes extrayendo las conclusiones más relevantes.

\section{ANÁLISIS POSTURAL}

Con el fin de monitorizar la postura del usuario de forma adecuada, en esta sección se realiza un análisis postural que permita detectar los puntos más relevantes donde se colocarán los sensores de fuerza FSR.

Con el objetivo de realizar este análisis, se han seleccionado 12 posturas que se dan con frecuencia durante la vida diaria [15] (Tabla 1). En la figura 1 se muestra una representación visual de cada una de estas posturas, de cara a facilitar la comprensión de las mismas.
Tabla 1: Numeración de posturas de sedestación

\begin{tabular}{|c|l|}
\hline No & Postura \\
\hline 1 & Postura correcta \\
2 & Inclinación hacia la derecha \\
3 & Inclinación hacia la izquierda \\
4 & Inclinación hacia adelante \\
5 & Inclinación hacia atrás \\
6 & Pierna derecha cruzada sobre izquierda \\
7 & Pierna izquierda cruzada sobre derecha \\
8 & Postura 6 + Glúteos adelante \\
9 & Postura $7+$ Glúteos adelante \\
10 & Hipercifosis \\
11 & Sentarse en el borde del asiento \\
12 & Borde del asiento, espalda atrás \\
\hline
\end{tabular}

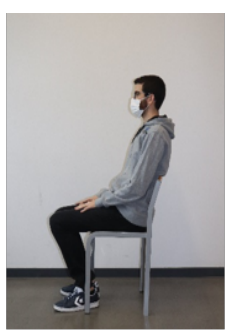

1

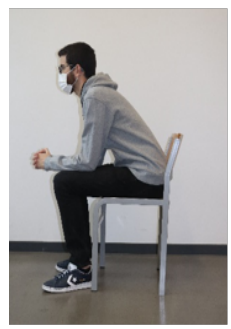

4

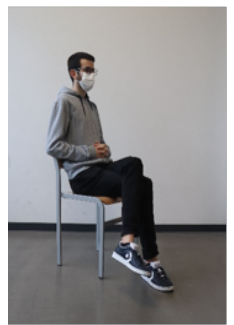

7

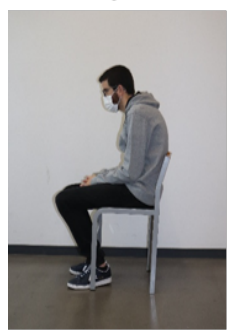

10

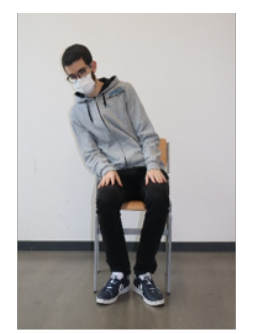

2

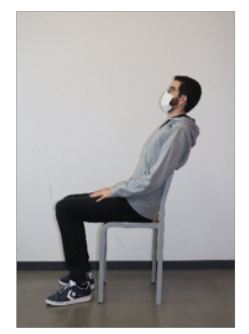

5

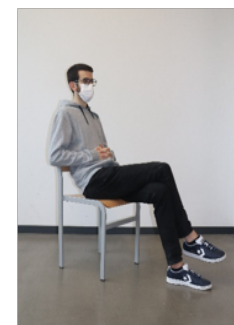

8

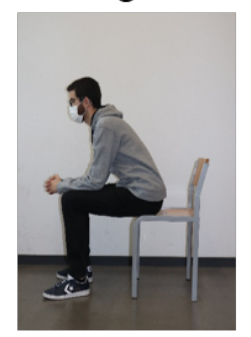

11

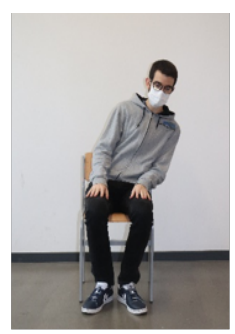

3

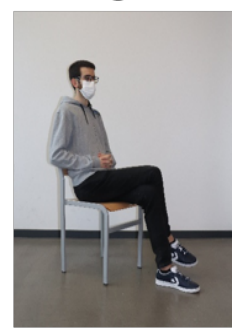

6

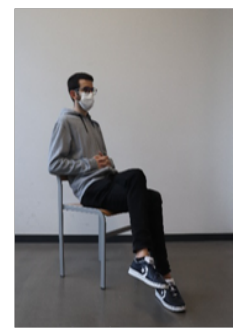

9

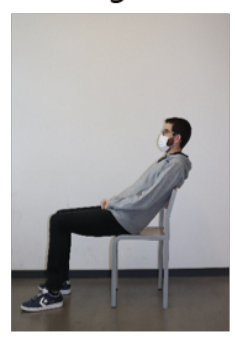

12
Figura 1: Representación visual de posturas de sedestación objeto de estudio 
Para la selección de los puntos significativos, se han realizado ensayos con diferentes personas sanas y jóvenes en las 12 posturas de sedestación detalladas en la tabla 1. Para monitorizar los ensayos, se han usado dos mallas comerciales Seating Mat Dev Kit de Sensing Tex que se han colocado tanto en el asiento como en el respaldo una silla. Se trata de un mallado comercial, compuesto por 256 sensores de presión distribuidos en una matriz de 16x16 [17]. Estas mallas vienen equipadas con su propio software de interpretación de datos que se utiliza para la selección de puntos de interés.

Una vez realizado los ensayos, para cada una de estas posturas, y mediante el software de la malla comercial, se han determinado los puntos más significativos. Se consideran como puntos significativos, por una parte, aquellos puntos en los que la presión ejercida aumenta con respecto a la postura correcta (Postura 1), y por otro lado, aquellos puntos donde la presión disminuye en gran medida con respecta a la postura correcta.

Este software representa mediante una escala de colores el rango de presiones que se miden en ella, dibujando en azul los puntos donde menor presión se detecta, posteriormente en verde las zonas con una presión superior, para acabar con un amarillo y naranja en las zonas donde más presión se ejerce.

A continuación, se muestran alguna de estas posturas para ilustrar el procedimiento llevado a cabo, donde se han representado mediante cruces rojas aquellos puntos donde la presión es más significativa para esa postura, y mediante puntos verdes, aquellos puntos donde se detecta una ausencia de presión con respecto a la postura correcta.

El punto de partida es la denominada postura correcta (Postura 1). Se trata de la postura que se desea que el usuario mantenga durante una mayor parte del tiempo, con ligeras modificaciones para evitar la aparición de úlceras.

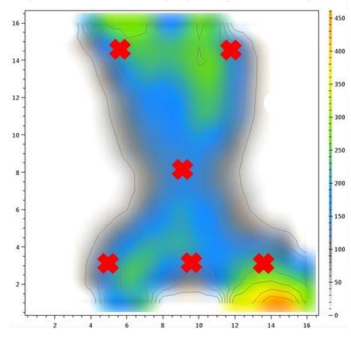

(a) Respaldo

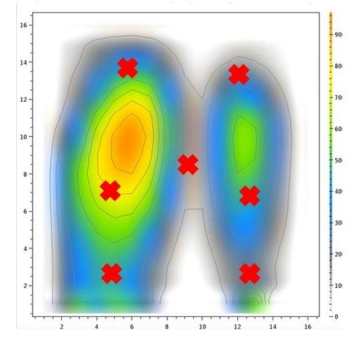

(b) Asiento
Figura 2: Distribución de presiones Postura 1: Postura correcta

En las figuras 2a y 2b se representa la distribución de presiones de respaldo y asiento en la posición correcta (Postura 1). Tal y como se aprecia la es- palda se mantiene recta, con los hombros echados hacia atrás, y apoyado sobre la zona lumbar. Por lo general, se trata de una postura en la que no se alcanzan grandes distribuciones de presión sobre el respaldo. Además, en el asiento, se distribuye el peso entre ambas caderas, con tendencia a apoyar más sobre un muslo que en otro. Esta postura no aporta excesiva información de la localización donde se tienen que ubicar los sensores, dado que la distribución de peso es uniforme a lo largo de las superficies de apoyo, pero sirve como base de partida para el posterior análisis del resto de posturas.

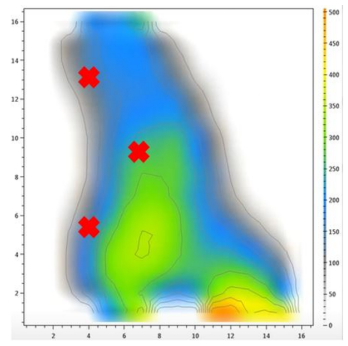

(a) Respaldo

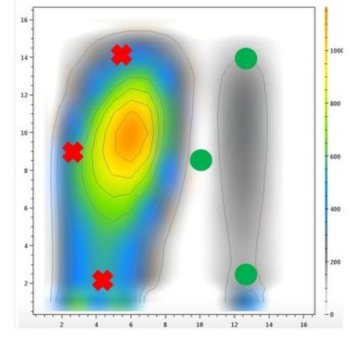

(b) Asiento
Figura 3: Distribución de presiones Postura 2: Desplazamiento lateral.

Por otra parte, en las figuras 3a y 3b se muestra la distribución de presiones para la simulación de una caída lateral (Postura 2). De estos ensayos, se deduce que se produce un aumento de presión en la zona de los muslos hacia el lado donde se está realizando la caída, mientras que se reducen en el lateral opuesto. Es necesario, colocar sensores en los laterales del asiento, que permitan monitorizar esos desplazamiento laterales. Estos sensores laterales deben colocarse a la altura de los isquiones, para monitorizar la zona donde se acumula la mayor presión sobre el asiento.

En el respaldo, se produce un comportamiento similar, los puntos de presión iniciales se encuentran en el centro del respaldo. A medida que se desplaza la espalda, los puntos de presión se concentran en el lateral hacia donde se está produciendo la caída. Además, es necesario que estos sensores laterales se encuentren situados a diferentes alturas, puesto que a medida que esta inclinación se hace más pronunciada, el peso se concentra en la esquina inferior izquierda del cuadrante. Esta diferencia de alturas, permite conocer el grado de inclinación de la caída. Es imprescindible, por tanto, disponer de al menos dos sensores a cada lado, dispuestos a diferentes alturas para monitorizar estos desplazamientos laterales.

Al igual que se analizan las caídas laterales, se hace un estudio de los desplazamientos frontales (Postura 4). Para ello, en las figuras 4a y 4b, se 


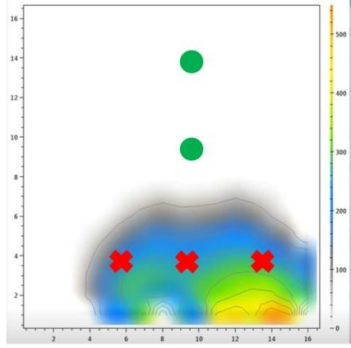

(a) Respaldo

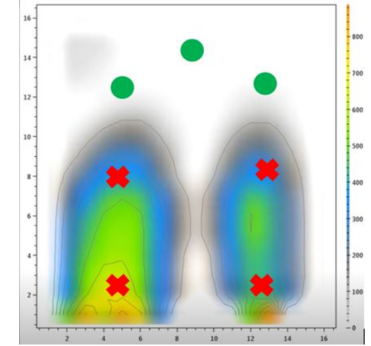

(b) Asiento
Figura 4: Distribución de presiones Postura 4: Inclinación hacia adelante.

representan la distribución de presiones obtenida al realizar una inclinación frontal. En estas distribuciones se observa como el participante deja de realizar presión (representado mediante puntos verdes en la imagen) a lo largo de los glúteos y ésta se traslada a las piernas, concentrándose a la altura de los bíceps femorales. En consecuencia, es necesario monitorizar esta variación de presión mediante la colocación de sensores a la altura de los isquiones y coxis.

En cuanto al respaldo, la presión se concentra en la zona inferior del respaldo. Es necesario mantener al menos un sensor en esa zona lumbar, que permita distinguir una inclinación frontal y una ausencia de contacto en el respaldo.

\section{DISPOSITIVO DE MONITORIZACIÓN POSTURAL}

A partir del análisis realizado en el apartado anterior, y extendido para el resto de posturas, se ha concluido que para la monitorización de las 12 posturas definidas es suficiente con el uso de 8 sensores en el asiento y 8 sensores en el respaldo, sumando un total de 16 sensores de fuerza distribuidos de forma discreta.

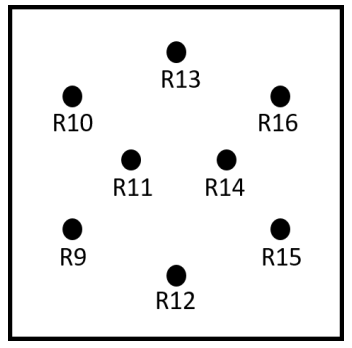

(a) Sensores respaldo

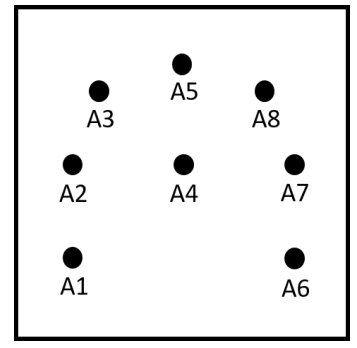

(b) Sensores asiento
Figura 5: Distribución de sensores del Dispositivo de Monitorización Postural

El diseño del respaldo se muestra en la figura 5a. La mayor parte de los sensores se disponen en la zona central, para la monitorización de la espina dorsal. Se disponen a diferentes alturas para monitorización de diversas partes de la espalda. De esta forma, el sensor R12 se encarga de monitorizar la lumbar, los sensores R11 y R14 la zona dorsal inferior y el sensor R13 la zona dorsal superior y cuello. Además, tal y como se ha comentado, se disponen de cuatro sensores adicionales, dos a cada lado, para la monitorización de desplazamientos laterales. Estos sensores son, concretamente, los sensores R9 y R15 para monitorizar las desviaciones sobre la zona lumbar y los sensores R10 y R16 para monitorizar las inclinaciones a la altura de los omoplatos.

El diseño del asiento se muestra en la figura 5b. Los sensores A1 y A6 están ubicados de forma que se lleve a cabo la monitorización de los bíceps femorales, y conjuntamente con el sensor A4, situado sobre el perineo, permite la detección de desplazamientos frontales sobre el asiento. Los sensores A2 y A7, ubicados sobre los glúteos mayores, junto con los sensores A3 y A8, colocados sobre los isquiones, permiten monitorizar los desplazamientos tanto laterales como longitudinales. Por último, el sensor A5, permite monitorizar la zona junto al coxis.

Con la localización de los sensores definida, se ha diseñado un prototipo de bajo coste para monitorización postural, utilizando para ello 16 sensores FSR 400 del fabricante Intelink Electronics [9]. La adquisición de los datos de estos sensores se ha realizado mediante una placa de control inalámbrica Arduino MEGA 2560, alimentada por una batería externa que proporciona un uso superior a las 24 horas. De esta forma, los datos se envían a través de bluetooth a un ordenador externo, donde se aloja una interfaz intuitiva que representa en tiempo real la presión ejercida sobre cada uno de los sensores, facilitando la visualización.

\section{VALIDACIÓN EXPERIMENTAL}

Para validar el diseño del prototipo propuesto, se han llevado a cabo ensayos experimentales con personas sanas, a los que se les ha vuelto a pedir que realizaran cada una de las posturas definidas en la tabla 1. Por tanto, se trata de un ensayo similar al anterior, pero en esta ocasión, además del uso de las mallas comerciales Seating Mat Dev Kit, se hace uso del prototipo de monitorización postural basado en sensores FSR. De esta forma, se puede realizar un análisis comparativo entre ambos sistemas de medición, permitiendo así, demostrar la efectividad del método de monitorización postural de bajo coste propuesto. 
De cara a analizar la validación realizada, se presentan a continuación en más detalle la comparativa realizar para las posturas 1, 5, 4 y 3 definidas en la tabla 1.

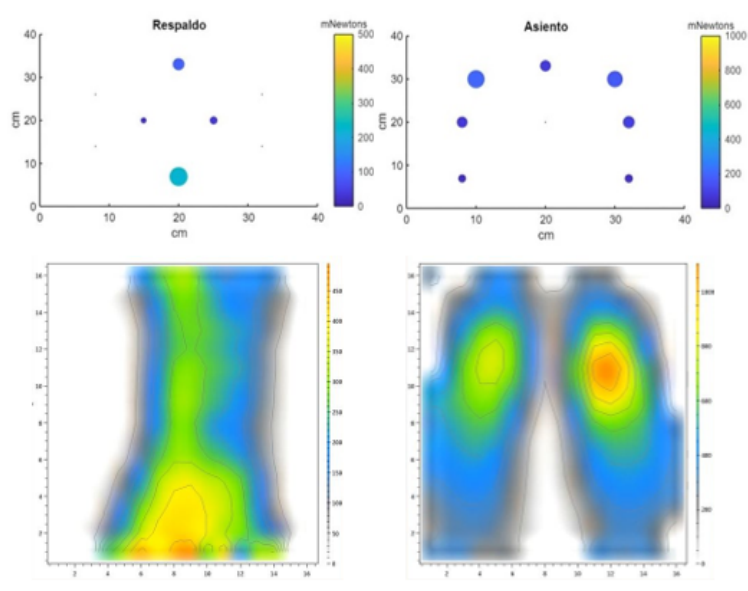

Figura 6: Postura 1: Postura correcta

En la figura 6 se muestran los resultados de la postura correcta (Postura 1). En la zona superior de la figura se representan los resultados obtenidos mediante el prototipo propuesto, y en la zona inferior la distribución de presiones obtenida mediante las mallas de presión comerciales. Para facilitar la interpretación de los datos obtenidos mediante el prototipo presentado, se ha incluido una variación en tamaño de los puntos mostrados, proporcional a la fuerza aplicada. Además, se aplica una escala de colores que abarca desde un azul oscuro para las fuerzas más bajas, hasta un amarillo para las mayores fuerzas medidas.

En la postura correcta, tal y como se ha explicado previamente, se mantiene la espalda recta apoyada en el respaldo. Esta rectitud se ve plasmada en el dispositivo presentado, distribuyéndose el peso sobre los cuatro sensores del respaldo situados en el eje de simetría, R11, R12, R13 Y R14. Además, reparando la atención a la magnitud de las presiones, se puede concluir que corresponden con las medidas tomadas por la malla comercial, e indican cierto arqueamiento de la columna, siendo la zona lumbar donde mayor fuerza se realiza.

Por otra parte, el peso se distribuye uniformemente a lo largo de los sensores del asiento. Se observa, cómo la ubicación de los sensores A3 y A8 se ha hecho de forma óptima, ya que, se buscaba colocarlos sobre los isquiones, zona donde se producen mayores fuerzas. Tal y como se comprueba, esta zona se ha caracterizado correctamente mediante estos sensores. El resto de sensores, van disminuyendo la fuerza medida a medida que se alejan de estos dos puntos.

En la figura 7, se presentan los resultados obteni-
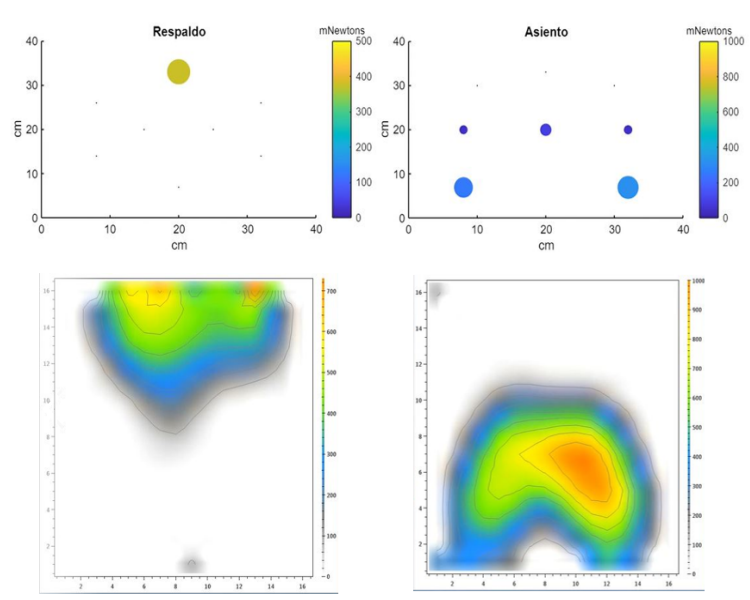

Figura 7: Postura 5: Inclinación hacia atrás

dos para la Postura 5. En esta postura, se inclina la espalda hacia atrás, apoyando el peso en la zona superior del respaldo, aumentándose por tanto, la fuerza medida en esa zona. Además, para facilitar esta inclinación, se desplazan los glúteos en el asiento ligeramente hacia adelante.

Esta postura, queda representada correctamente mediante los sensores discretos. En el asiento, existe un desplazamiento de la fuerza hacia la mitad delantera, mientras que la zona trasera se queda sin presión. La colocación de los sensores A3, A5 y A8, que permiten percibir esta ausencia de fuerza, conjuntamente con el sensor A2, A4 y A7 permiten caracterizar el desplazamiento en el asiento. La magnitud de este último grupo de sensores permite, además, cuantificar el grado de desplazamiento, llegando a una situación en la que no devuelven ningún valor de fuerza. Se trata de la situación en la que el usuario realiza la postura 12 de la tabla 1, y está completamente reclinado hacia adelante.

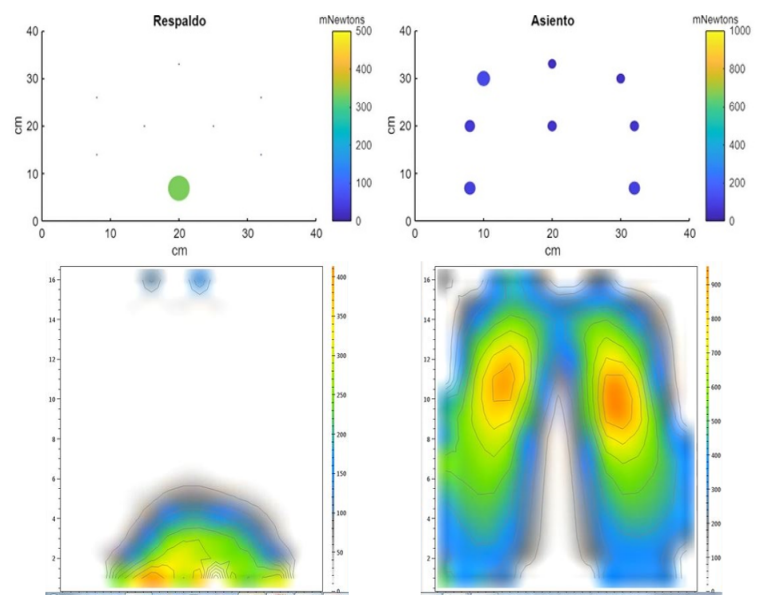

Figura 8: Postura 4: Inclinación hacia adelante

La caracterización del asiento de la postura 4, representada en la figura 8 , es similar a la de la pos- 
tura correcta, con ligeros cambios. Se mantiene una distribución uniforme a lo largo de todo el asiento, pero en esta ocasión la fuerza ejercida sobre los sensores A3 y A8 no es tan pronunciada, dado que el centro de masas de la espalda se desplaza hacia adelante. Esta es la misma razón, por la cual, el sensor A4, colocado a la altura del perineo y que en la postura correcta no realiza ninguna lectura, pasa a medir fuerzas. Este sensor sirve, por lo tanto, como indicador para monitorizar la inclinación delantera de la espalda.

La información del asiento viene acompañada por la del respaldo, donde el único sensor que mide fuerza es el R12 sobre la zona lumbar. Se recalca la importancia de la ubicación de este sensor, puesto que, de no ubicarse ahí, no se podría diferenciar un desplazamiento de los glúteos de una inclinación frontal de la espalda.

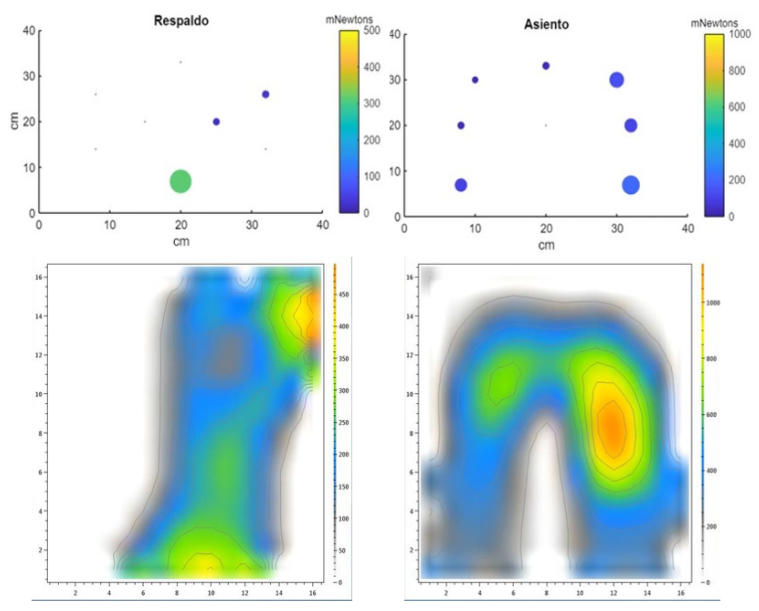

Figura 9: Postura 3: Inclinación hacia la izquierda

En último lugar, en la figura 9 se presentan los resultados para la Postura 3. En esta postura, se pretende caracterizar un desplazamiento hacia la izquierda de la espalda, para prevenir caídas laterales. La curvatura de la espalda queda perfectamente definida por los sensores R12, R14 y R16, quedando definida la dirección del desplazamiento. Además, mediante el sensor R15, situado en la zona lumbar izquierda, se puede monitorizar cuan pronunciada es la inclinación realizada.

En cuanto a la situación del asiento, la posición de los glúteos no varía con respecto a la posición correcta. Sin embargo, a medida que se inclina la espalda hacia una lateral, el centro de masas de estas se modifica, y queda reflejado en el asiento, mediante un aumento generalizado de las fuerzas medidas mediante los sensores de dicho lado.

Resumiendo, tras un estudio individualizado para cada una de las 12 posturas definidas, se puede concluir que cada una de ellas queda correctamente caracterizada por 16 sensores dispuestos en po- siciones relevantes identificadas en este artículo. Por lo tanto, se concluye que es posible monitorizar estas posturas sin necesidad de hacer uso de un elevado número de sensores, consiguiendo simplificar el dispositivo de medición, reduciendo los costes y aumentando su autonomía.

\section{CONCLUSIONES}

En este trabajo, se ha presentado un dispositivo de bajo coste para monitorizar la postura en sedestación, enfocado a personas de la tercera edad. En este dispositivo se hace uso de sensores de fuerza, dejando al margen las mallas de presión.

Con el fin de reducir el número de sensores utilizados, pero sin que ello repercuta en una pérdida de información, se ha realizado un análisis postural con personas jóvenes y sanas. Este análisis ha permitido definir un total de 16 puntos relevantes, 8 en el asiento y 8 en el respaldo.

Sobre estos puntos, se ha desarrollado un primer prototipo compuesto por 16 sensores FSR ubicados en los puntos óptimos previamente identificados. Mediante la realización de nuevos ensayos, se ha validado el diseño propuesto y se ha concluido que el conjunto de puntos seleccionados permite caracterizar de forma adecuada cada una de las posturas definidas.

Por lo tanto, el dispositivo de monitorización postural propuesto permite llevar un seguimiento eficiente, en tiempo real, del estado postural de los pacientes, reduciendo el coste del hardware, así como aumentando su autonomía. Además, al trabajar con un número menor de datos, se simplifica el almacenamiento y análisis de los mismos. Esto, permitirá dar soporte a los especialistas sanitarios, proporcionándoles información objetiva del estado de los pacientes, así como detectar patrones de comportamiento, situaciones anómalas y caídas.

En trabajos futuros se pretende seguir trabajando en la linea de monitorización y diagnóstico postural. Así, se pretenden realizar ensayos experimentales de mayor duración, que permitan entre otras cosas, comprobar la robustez del sistema ante cambios de temperatura. Además, se pretende incorporar técnicas inteligentes que permitan la detección de posturas, así como patrones de comportamiento postural de los usuarios, que permitan dar idea del estado de éstos, todo ello mediante las mediciones realizadas con el dispositivo propuesto.

\section{Agradecimientos}

Este trabajo ha sido financiado por: FEDER/Ministerio de Ciencia e Innovación - Agencia Estatal de Investigación/Proyecto PID2020- 
112667RB-I00, la Universidad del Páis Vasco UPV/EHU (GIU19/045), así como por el contrato predoctoral PRE-2020-1-0073 del Gobierno Vasco.

\section{English summary}

POSTURAL MONITORING DEVICE FOR ELDERLY WITH A HIGH DEGREE OF SEDENTARISM

\begin{abstract}
Postural monitoring in the elderly is essential to avoid the appearance of musculoskeletal problems, as well as to prevent risky situations and falls. In this work, a monitoring device composed of 16 FSR (Force Sensitive Resistor) sensors is presented, discreetly placed along the seat and back of a chair. The selection of the location of these sensors has been carried out through a previous study, where the most significant points have been identified. To validate the monitoring device, different experimental tests have been carried out where the results obtained by the proposed device have been compared with those measured with a pressure mat. The results show that the positions presented are correctly characterized by the proposed system, being able to reduce the cost of the hardware and increase its autonomy.
\end{abstract}

Keywords: Postural Analysis; Monitoring; Sedentary lifestyle; Force Sensors.

\section{Referencias}

[1] A. Abellán García, P. Aceituno Nieto, J. Pérez Díaz, D. Ramiro Fariñas, A. Ayala García, and R. Pujol Rodríguez. Un perfil de las personas mayores en españa, 2019. indicadores estadísticos básicos. Informes Envejecimiento en red, (22):38, 2019.

[2] A. Abobakr, M. Hossny, and S. Nahavandi. A skeleton-free fall detection system from depth images using random decision forest. IEEE Systems Journal, 12(3):2994-3005, 2018.

[3] B. Arnrich, C. Setz, R. La Marca, G. Tröster, and U. Ehlert. What does your chair know about your stress level? IEEE Transactions on Information Technology in Biomedicine, 14(2):207-214, 2010.

[4] A. Biswas, P. I. Oh, G. E. Faulkner, R. R. Bajaj, M. A. Silver, M. S. Mitchell, and D. A. Alter. Sedentary time and its association with risk for disease incidence, mortality, and hospitalization in adults a systematic review and meta-analysis. Annals of Internal Medicine, 162(2):123-132, 2015.

[5] S. Chopra, M. Kumar, and S. Sood. Wearable posture detection and alert system. Proceedings of the 5th International Conference on System Modeling and Advancement in Research Trends, SMART 2016, pages 130-134, 2017.

[6] A. Cristina, F. Geraldo, and A. M. Kuasne. Prototype of Wearable Technology Applied to the Monitoring of the Vertebral Column. International Journal of Online and Biomedical Engineering, 16(01):34-50, 2020.

[7] A. Edjolo, C. Proust-Lima, F. Delva, J.F. Dartigues, and K. Pérès. Natural history of dependency in the elderly: a 24-year population-based study using a longitudinal item response theory model. American journal of epidemiology, 183(4):277-285, 2016.

[8] B. El-Sayed, N. Farra, N. Moacdieh, H. Hajj, R. Haidar, and Z. Hajj. A novel mobile wireless sensing system for realtime monitoring of posture and spine stress. 2011 1st Middle East Conference on Biomedical Engineering, MECBME 2011, pages 428-431, 2011.

[9] I. E. FSR 400. Available online: https://www.interlinkelectronics.com/fsr400, accessed 2021-06-02.

[10] M. Kim, H. Kim, J. Park, K. K. Jee, J. A. Lim, and M. C. Park. Real-time sitting posture correction system based on highly durable and washable electronic textile pressure sensors. Sensors and Actuators, A: Physical, 269:394-400, 2018.

[11] G. Liang, J. Cao, and X. Liu. Smart cushion: A practical system for fine-grained sitting posture recognition. In 2017 IEEE International Conference on Pervasive Computing and Communications Workshops, PerCom Workshops 2017, pages 419-424, 2017.

[12] B. Liu, Y. Li, S. Zhang, and X. Ye. Healthy human sitting posture estimation in RGB-D scenes using object context. Multimedia Tools and Applications, 76(8):10721-10739, 2017. 
[13] C. Ma, W. Li, R. Gravina, J. Cao, Q. Li, and G. Fortino. Activity level assessment using a smart cushion for people with a sedentary lifestyle. Sensors (Switzerland), 17(10):1-19, 2017.

[14] J. Meyer, B. Arnrich, J. Schumm, and G. Troster. Design and modeling of a textile pressure sensor for sitting posture classification. IEEE Sensors Journal, 10(8):13911398, 2010.

[15] B. Mutlu, A. Krause, J. Forlizzi, C. Guestrin, and J. Hodgins. Robust, low-cost, nonintrusive sensing and recognition of seated postures. UIST: Proceedings of the Annual ACM Symposium on User Interface Softaware and Technology, 4(1):149-158, 2007.

[16] H. Pazhoumand-Dar. FAME-ADL: a datadriven fuzzy approach for monitoring the ADLs of elderly people using Kinect depth maps. Journal of Ambient Intelligence and Humanized Computing, 10(7):2781-2803, 2019.

[17] S. S. Sensing Mat Dev Kit. Available online: http://sensingtex.com/product/seatingmat-dev-kit/, accessed 2020-02-24.

[18] M. Tremblay. Letter to the editor: Standardized use of the terms sedentary and sedentary behaviours. Applied Physiology, Nutrition and Metabolism, 37(3):540-542, 2012.

[19] P. D. United Nations, Department of Economic and Social Affairs. World Population Prospects: The 2019 Revision, Key Findings and Advance Tables. 2019.

[20] W. J. Yi, O. Sarkar, S. Mathavan, and J. Saniie. Wearable sensor data fusion for remote health assessment and fall detection. IEEE International Conference on Electro Information Technology, pages 303-307, 2014.

[21] Y. Zhang, S. Markovic, I. Sapir, R. C. Wagenaar, and T. D. Little. Continuous functional activity monitoring based on wearable tri-axial accelerometer and gyroscope. 2011 5th International Conference on Pervasive Computing Technologies for Healthcare and Workshops, PervasiveHealth 2011, pages 370-373, 2011. under the terms and conditions of the Creative Commons Attribution CC BY-NC-SA 4.0 license (https://creativecommons.org/licenses/by-ncsa/4.0/deed.es).

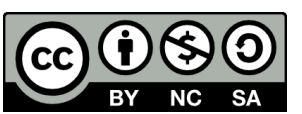

C) 2021 by the authors.
Submitted for possible
open access publication 